\title{
Vers une plateforme sémantique pour l'enseignement des sciences et de la culture numérique
}

Thibaud Hulin

\section{QpenEdition}

\section{Journals}

Édition électronique

URL : http://journals.openedition.org/trema/3379

DOI : 10.4000/trema.3379

ISSN : 2107-0997

Éditeur

Faculté d'Éducation de l'université de Montpellier

Édition imprimée

Date de publication : 1 mai 2016

Pagination : $37-49$

ISBN : 979-10-96627-00-4

ISSN : 1167-315X

Référence électronique

Thibaud Hulin, «Vers une plateforme sémantique pour l'enseignement des sciences et de la culture numérique », Tréma [En ligne], 44 | 2016, mis en ligne le 01 juin 2016, consulté le 19 avril 2019. URL http://journals.openedition.org/trema/3379; DOI : 10.4000/trema.3379

Ce document a été généré automatiquement le 19 avril 2019

Trema 


\title{
Vers une plateforme sémantique pour l'enseignement des sciences et de la culture numérique
}

\author{
Thibaud Hulin
}

\section{Introduction}

1 Quelles sont les conditions favorables à la mise en œuvre d'une formation numérique prometteuse et réussie? S'il est prétentieux d'apporter une réponse purement technologique à cette question, il est aussi risqué de prétendre ignorer la question du choix des outils, de leur conception et de leurs usages possibles. Notre position est donc plutôt d'envisager le croisement entre les innovations technologiques et les innovations éducatives. Les possibilités du web sémantique étant le plus souvent méconnues des chercheurs en éducation ou en formation, nous voudrions ici montrer l'intérêt de joindre de façon pluridisciplinaire la réflexion sur les outils à la réflexion sur la pédagogie. À l'heure du développement intense de l'enseignement numérique, les chercheurs en éducation ne peuvent plus ignorer les problèmes informatiques qui y sont liés, et réciproquement. Ainsi nous observons la multiplication des plateformes et des ressources pédagogiques, des systèmes de gestion de l'apprentissage (type Moodle) et des MOOCs ${ }^{1}$. Cependant, dans la mesure où ces plateformes sont hétérogènes et les ressources encore rarement mutualisées, leur coût d'entretien est assez important. Une fois qu'elles ont répondu au besoin qui les a fait naitre, elles risquent de tomber dans l'oubli peu de temps après leur lancement. De plus, ces bases de documents ne savent pas communiquer entre elles, ce qui rend ces initiatives isolées : une plateforme ne peut pas indiquer l'existence d'un document disponible sur une autre plateforme alors même qu'il répond au besoin de l'utilisateur. Enfin, le vocabulaire des requêtes et des métadonnées qui décrivent les ressources de la plateforme n'est pas nécessairement le même que celui de l'utilisateur qui risque de passer à côté du document dont il a besoin. Dès lors, comment envisager un «langage commun » entre ces différentes plateformes qui puisse unifier chercheurs en éducation ou en informatique, formateurs, éducateurs, et apprenants? Comment assurer 
la pérennité, l'accessibilité et la visibilité d'une base pédagogique ? Comment produire des contenus numériques qui répondent clairement aux besoins des usagers? Comment éviter que les plateformes d'enseignement à distance ne tombent dans l'oubli faute de maintenance? Or, il existe des réponses technologiques à ces besoins: ce sont les technologies du "web sémantique». Pour autant, cette approche ne peut être que l'affaire des informaticiens. Car pour décrire les documents pédagogiques à l'aide d'un langage partagé, il nous faut décrire l' "univers discursif » dans lequel nous situons nos recherches, l'ensemble des concepts clés de l'apprentissage et de leurs relations. Pour répondre à cette problématique, nous proposons dans cet article un programme de recherche visant à mettre en patrimoine les situations d'apprentissage. Après avoir décrit l'intérêt et le fonctionnement des technologies sémantiques pour l'éducation, nous présenterons notre projet de plateforme sémantique pour l'enseignement des sciences et de la culture numérique. Le résultat de notre travail concerne d'une part un programme pour la réalisation d'une plateforme sémantique, d'autre part une méthode pour représenter les situations d'apprentissage à partir de l'activité cognitive des apprenants. Bien que ce programme concerne l'enseignement des sciences en général, nous exposerons ici l'approche pédagogique développée au sujet des usages du numérique.

\section{Mise en patrimoine des situations d'apprentissage}

2 Pour répondre au risque d'isolement des bases de données éducatives, nous proposons un objectif général de mise en patrimoine des situations d'apprentissage, c'est-à-dire de partage des ressources autour d'un vocabulaire commun. Pour L'UNESCO (2003), le patrimoine culturel et immatériel désigne : «les pratiques, représentations, expressions, connaissances et savoir-faire - ainsi que les instruments, objets, artefacts et espaces culturels qui leur sont associés - que les communautés, les groupes et, le cas échéant, les individus reconnaissent comme faisant partie de leur patrimoine culturel ». Ainsi la mise en patrimoine des situations d'apprentissage structure les communautés éducatives. Cela consiste à rassembler les concepts permettant de décrire et de penser les situations d'apprentissage et de formation, médiées informatiquement ou non, pour produire un socle pédagogique et épistémologique commun, c'est-à-dire un langage partagé. Ce socle permet de développer une plateforme expérimentale pour articuler des ressources hétérogènes, déposées sur des dépôts numériques divers. Il est destiné animer la formation, l'enseignement, l'apprentissage et l'étude scientifique des situations d'apprentissage.

3 Nous postulons que la mise en patrimoine des situations d'apprentissage est en mesure de structurer les communautés éducatives. Cette approche vise à rassembler les concepts permettant de décrire et de penser les situations d'apprentissage et de formation, médiées informatiquement ou non, pour produire un socle pédagogique et épistémologique commun, sous la forme d'un vocabulaire partagé. Sur le plan des techniques, ce socle peut s'appuyer sur les technologies du "web sémantique » (BernersLee, Hendler et Lassila, 2001), appelé aussi «web 3.0 » par opposition au web 2.0 qui désigne le web des blogs et des réseaux sociaux. Le web sémantique regroupe un ensemble de technologies et de formats (RDF, SPARQL, SKOS, FOAF, OWL...) qui visent à standardiser les échanges de données afin que différentes bases puissent communiquer entre elles via un " langage » commun (un vocabulaire et des règles de grammaire). Ces technologies et normes visent aussi à décrire les données elles-mêmes. Ainsi un 
«contact », un «formateur » et un «auteur » peuvent être décrits à l'aide du même langage commun à l'aide de la norme $\mathrm{FOAF}^{2}$. Par exemple, une recherche dans un site web spécialisé en sciences de l'éducation avec le mot-clé «Charlot» doit pouvoir déboucher sur les ouvrages du pédagogue et chercheur Bernard Charlot, et non pas sur le personnage de cinéma (ou alors secondairement). Pour cela, il faut que les machines sachent décrire au niveau sémantique les données, et non pas se contenter de repérer des proximités syntaxiques entre requêtes et résultats (des ressemblances entre mots). Nous avons besoin de dire que Charlot est le nom d'un chercheur, que ce chercheur a une propriété d'être chercheur en sciences de l'éducation, qu'un chercheur est une personne, enfin que les sciences de l'éducation sont une discipline. Si cela paraît évident à un humain en raison de ses références culturelles, il est nécessaire de décrire explicitement ces liens à une machine pour qu'elle puisse comprendre notre requête. À côté des documents pédagogiques rassemblés, nous avons alors à construire non pas une base de données relationnelle, ce qui nous restreint au web syntaxique, mais une ontologie, qui est son équivalent porté au niveau du web sémantique.

4 Le mot ontologie au sens informatique du terme est une représentation de l'univers sémantique d'un discours : elle décrit les relations entre des concepts, par exemple dans : un chercheur est une personne, ou encore : l'activité est un concept central des dites théories de l'activité. Cette base de connaissance est définie par Gruber (1993, p. 199) comme un « ensemble de spécifications formelles explicites de termes d'un domaine et de relations entre elles». L'ontologie relève du vocabulaire contrôlé: elle fournit une description d'individus : des concepts, des personnes, des ressources uniques... Ceux-ci appartiennent à des classes et des sous-classes, par exemple la classe des auteurs ou celle des disciplines. Individus et classes admettent des descripteurs qui leur sont propres, appelés attributs, comme dans " un chercheur peut être publiant » ou bien des relations avec d'autres classes ou individus, comme dans "un chercheur a une discipline ", où « chercheur » et « discipline » ont une relation de type « possède une ».

5 Comme on le voit, l'écriture d'ontologies force à expliciter et à clarifier les concepts et leurs relations d'un vocabulaire dans le but de partager et d'éviter toute ambiguïté. Le choix du recours aux ontologies est ainsi justifié par l'objectif d'une accessibilité améliorée aux documents pédagogiques: la communication entre les bases en est facilitée. Le vocabulaire employé pour décrire les ressources est indépendant du vocabulaire employé par l'utilisateur qui formule une requête.

6 Prenons l'exemple d'une ressource décrite comme étant « une ressource en didactique de la physique». Si un enseignant recherche une ressource en "enseignement de la physique ", il ne la trouvera pas car les mots-clés employés pour la requête ne sont pas les mêmes que ceux pour décrire la ressource. En revanche, si l'ontologie indique de «la didactique et l'enseignement sont des démarches pédagogiques", alors la machine pourra présenter à l'enseignant la ressource en question. Il est enfin possible d'effectuer des calculs par la machine pour produire de nouvelles connaissances en produisant des requêtes complexes, par exemple: «donne-moi la liste des auteurs au programme avec lesquels je puisse enseigner l'énergie ». Il est aussi possible d'accéder à des définitions (possibles ou consensuelles) pour l'utilisateur de la plateforme. Finalement, pour produire des ontologies pour l'éducation, il est nécessaire de décrire différents types de connaissances :

- les savoirs disciplinaires du domaine avec les concepts importants et leurs relations ; 
- les ressources documentaires hétérogènes (vidéos, images, textes, références bibliographiques, documents scénarisés, Serious Games, portails, etc.) ;

- les activités pédagogiques en présentiel et en ligne (pour la formation, la recherche, l'enseignement) ;

- les compétences (du domaine à enseigner, les compétences transversales comme celles qui relèvent de la littératie numérique) ;

- les situations et contextes d'apprentissage ;

- les acteurs : apprenants, enseignants, formateurs de formateurs, chercheurs) ;

- les organisations (écoles, universités, etc.).

7 Ainsi, ces différents types de connaissances constitueront autant d'ontologies de domaines. Il devient alors possible de concevoir des parcours scénarisés dans l'ensemble des ontologies en conseillant des ontologies en fonction du profil de l'apprenant, du formateur ou de l'enseignant.

8 À titre d'exemple, la NASA a proposé un ensemble d'ontologies baptisé SWEET (Raskin et Pan, 2005) ${ }^{3}$ afin de réunir et d'unifier leurs données en science de la terre. Du côté des sciences de l'éducation et de la formation, il existe différents outils et standards, par exemple les normes SCORM ${ }^{4}$ et $\mathrm{LOM}^{5}$, pour structurer des documents pédagogiques. Par ailleurs, Dicheva, Sosnovsky, Gavrilova et Brusilovsky (2005) ont proposé un état de l'art des problèmes de recherche concernant la production d'ontologies pour les sciences de l'éducation. Ils concernent : le développement d'ontologies, les problèmes théoriques, les perspectives technologiques, les perspectives d'application. Pour ce qui concerne cet article, l'objectif général de mise en patrimoine des situations d'apprentissage concerne principalement les perspectives d'application: production d'une plateforme sémantique expérimentale, création de scénarios pédagogiques, production d'ontologies génériques liées au champ de l'apprentissage (activités, compétences, utilisateurs et groupes, organisations, concepts, situations), et ontologies de domaine. Une fois ce programme décrit, se pose la question du mode d'entrée dans ces ontologies : comment relier les situations d'apprentissage aux représentations sémantiques? Quels concepts retenir? Nous nous intéressons ici particulièrement au cas de la culture numérique.

\section{Modéliser la culture numérique}

Afin de réaliser une plateforme sémantique pour l'enseignement des sciences, la place de la culture numérique est essentielle puisqu'elle vise à permettre et à assurer les usages de la plateforme par des enseignants, des formateurs et des apprenants qui ne sont pas nécessairement des informaticiens. Nous recherchons ici des éléments pour construire une ontologie, c'est-à-dire un modèle de la culture numérique qui soit le plus durable possible.

Cependant, l'idée de formation à la culture numérique ne se réduit pas à l'usage d'un outil comme une suite bureautique. Ainsi nous distinguons formation procédurale et formation à la culture numérique. Dans une logique procédurale, on suit des recettes et on répète des procédures. Dans une logique culturelle, l'apprenant devient capable de s'approprier de nouveaux environnements informatiques. Avec Simondon (1969), nous pensons que la culture technique, laquelle nourrit la culture numérique, ne peut reposer sur un simple usage. La culture numérique doit ainsi pouvoir ainsi s'appuyer à la fois sur une culture technique, c'est-à-dire l'informatique, et sur une culture informationnelle, qui relève de 
l'usage d'un système d'information. L'enseignement de la culture numérique suppose donc de relier des littératies qui ont parfois été séparées : l'éducation aux médias, a recherche d'information documentaire, les usages du numérique ou la transmission de concepts informatiques dans une démarche non cloisonnée ; ce qui fait de l'enseignement de la culture numérique une démarche de type «translittératie » (Delamotte, Liquete et Frau-Meigs, 2014).

11 Cependant, la culture numérique s'appuie aussi sur l'histoire de l'activité numérique d'une personne. L'ensemble des cultures numériques d'une société donnée peut alors prétendre à constituer un patrimoine immatériel. Parce qu'il est vivant, ce patrimoine n'est pas formellement structuré : il intègre des participations, des remédiations et du bricolage (Deuze, 2006). La culture numérique, comme remédiation et comme constitution d'une identité numérique, relève du soin de soi (Stiegler, 2008) et d'une redéfinition instable, par exemple chez un blogueur. Cependant, comme participation et comme écriture de soi, elle fixe des éléments acquis (Foucault, 1994a), ce qui lui permet de constituer une mémoire durable voire un patrimoine collectif, dans la mesure où l'accès à Internet constitue un droit et un bien commun.

Pour construire une ontologie de la culture numérique, qui vise à coordonner un espace d'activité riche, vivant et complexe, différentes approches pédagogiques existent. L'approche procédurale ci-dessus évoquée, par les outils, a donné lieu à un ensemble de formations qui débouchent sur différentes certifications comme: le Passeport de compétences informatique européen ${ }^{6}$, le Test On Software Applications ${ }^{7}$ ou le Cambridge IT Skills Diploma ${ }^{8}$. Ces certifications visent à valider des niveaux de maîtrise de certains logiciels, en particulier de la suite bureautique de Microsoft. Pour utiles qu'elles soient sur le plan professionnel immédiat, ces formations débouchent nécessairement sur un enseignement de type procédural, centré sur l'usage d'un logiciel : elles impliquent une dépendance à l'égard de l'outil informatique choisi et de sa version. Les formations de type procédurales sont donc vite caduques puisque les outils évoluent rapidement. De leur côté, les certifications françaises de type Certificat de l'Informatique et d'Internet ${ }^{9}$ ont une approche différente centrée sur les compétences, plus centrée sur l'adaptativité des apprenants et sur leur culture numérique. Si le C2i permet «d'apprendre à apprendre » (Pelissier et Mailles-Viard Metz, 2012), il vise simplement à former à l'usage d'outils plutôt qu'à la " complexité de ses enjeux » (Beziat, 2012). Cette approche impose un découpage en domaines puis en compétences et donc en tâches, qui émiette l'activité réelle des apprenants. Enfin, elle fait aussi l'économie d'une justification, c'est-à-dire d'un modèle de l'activité humain/machine qui expliquerait la structure proposée. Elle repose sur l'expérience d'experts en fonction de leurs connaissances à un moment donné, non pas sur la logique interne, voire universelle et durable de l'activité numérique.

De leur côté, Bouchardon, Cailleau, Crozat et Bachimont (2011) ont tenté de construire l'apprentissage à partir d'une théorie des relations humain / machine. Ils ont développé une approche par les propriétés du numériques. Ils ont conçu une carte heuristique de ces propriétés. Ainsi la " clonabilité » est une propriété du numérique enseignable, et qui détermine, suppose-t-on, le copier-coller. L'avantage de cette approche est qu'elle propose un noyau de travail, basé sur une théorie de la relation humain / machine. Cependant, cette approche par les propriétés du numérique conduit à enseigner ou à utiliser en conception de formation des concepts de haut niveau d'abstraction, basé, comme pour le C2i, sur l'expertise. Leurs cartographes ont fait l'économie de la compréhension de l'activité et de l'expérience réelles d'utilisateurs non experts, laquelle 
repose sur une histoire à la fois individuelle et sociale qui n'est pas nécessairement et immédiatement " visible ». En ce sens, ils ont développé une approche experte, « macro », éloignée de l'activité cognitive humaine en tant qu'elle ne peut se comprendre qu'à un niveau «micro». Ils font fi des leçons des théoriciens de l'activité comme Vygotsky (1997), selon lequel l'histoire et l'historique sont présents dans l'activité située temporellement, ou Engeström (1999), qui montre la présence et l'influence des règles sociales dans l'activité individuelle de production numérique.

Ainsi, plutôt que de rechercher des «propriétés du numériques » qui déconnecteraient le technique de l'humain, comme si l'humain le contiendrait déjà, nous nous concentrerons sur la recherche de "schèmes d'action" (Piaget, 1972) lesquels, présentés en amont de l'activité pédagogique, joueront le rôle d'« organisateurs avancés " (Ausubel, 1960) afin d'effectuer des liens entre la culture acquise et la culture à développer de l'apprenant. À noter que la présentation d'une carte conceptuelle de ces « organisateurs avancés » aux apprenants est d'intérêt pédagogique (Willerman, 1991) ; à condition de bien définir ces organisateurs.

Le modèle "pédagogico-technique" recherché doit être un modèle centré sur les activités réelles des utilisateurs. Inspiré de la théorie des réseaux, il reprend le concept de couche issu du modèle OSI ${ }^{10}$ de Zimmermann (1980). Nous distinguons quatre couches du numériques qui vont de la machine vers le collectif humain, à partir duquel nous allons pouvoir asseoir les concepts de l'ontologie.

1. La couche matérielle du numérique détermine les objets numériques, qui sont des représentations mathématiques et formelles, manipulables et programmables (Manovitch, 2002).

2. La couche applicative dans laquelle apparaissent les fonctions des logiciels (comme artefacts), et qui structure en partie la culture numérique de l'apprenant. On distingue : le niveau fonctionnel, des possibilités d'interactions offertes par l'application; et le niveau médiatique du dispositif, fait de règles, de dits et de non-dits (Foucault, 1994b, p. 299).

3. La couche sémiotique selon laquelle un sujet, un être humain interprète les formes perçues de façon sensible à l'écran.

4. La couche politique dans laquelle s'institutionnalisent et se régulent les normes collectives ou communautaires de l'activité.

À l'intérieur de ce modèle, nous pouvons envisager quels sont les concepts mobilisés par une activité numérique. Par exemple, dans l'activité de rédaction d'un blog, on va rencontrer différents concepts généraux comme les traces numériques, les liens hypertextes, l'ergonomie web, etc. On utilisera ici le langage « ordinaire» de la culture numérique (copier-coller) et non pas un langage «expert» (clonabilité). Leur analyse permet les relations que certains concepts entretiennent avec d'autres, jusqu'à ce qu'ils deviennent spécifiques à l'activité singulière. Par exemple, on distinguera différents types de liens hypertextes (lien ancre, lien externe, etc.). Leur enseignement va alors vise à développer la créativité de l'utilisateur lorsqu'elle ou il se trouve face à un ensemble de règles ou contraintes: les contraintes liées à l'usage d'un ordinateur par rapport à un journal papier (couche matérielle), celles imposées par la conception du logiciel (couche applicative), celles qui tiennent de la perception de l'utilisateur (couche sémiotique), enfin celles qui relient un individu à différentes communautés ou sociétés (couche politique).

17 Selon cette approche, le développement d'une ontologie de la culture numérique recentrée sur l'activité permet de répondre aux besoins des utilisateurs en partant de 
l'activité réelle, grâce à un inventaire d'activités qui soient symptomatiques de l'activité pédagogique ou professionnelle visée. Mettre au centre du dispositif technique les structures fondamentales de l'activité humaine permet de retrouver ce croisement entre innovation technique et innovation éducative évoqué en introduction. Ainsi une telle ontologie ne peut être figée: ses mise à jour doivent refléter fondamentalement la manière dont activité technique et activité humaine s'entrecroisent et s'influencent mutuellement.

Tandis que l'approche par les outils ne peut être durable, que l'approche par les compétences introduit un certain arbitraire et que l'approche par les propriétés est trop abstraite, l'approche par les activités permet de décrire de façon fine l'activité numérique, à la fois technique et humaine, sans opposer fondamentalement ces pôles, mais sans les confondre non plus.

\section{Mise en œuvre et programme de formation}

En amont de la plateforme sémantique dont nous avons dessiné précédemment le programme, il nous faut nous assurer de la portée pédagogique de notre théorie des interactions humain/machine. Ainsi, nous avons réalisé différentes formations à la culture numérique auprès d'étudiants en suivant le plan décrit dans le tableau 1 . Certaines de ces formations à la culture numérique sont en ligne sur notre site personnel ${ }^{11}$. Ce dispositif active différentes couches de notre modèle théorique, abordées selon des étapes et un ordre contraint par les nécessités pédagogiques. Ces formations concernent différentes activités numériques : écriture collaborative, dans un réseau social, écriture multimédia, écriture hypertextuelle, etc. Les étudiants développent des compétences à partir des activités proposées qui peuvent être identifiées pendant l'observation. Ces compétences ne sont pas déterminées de façon a priori, dans l'activité prescrite: elles sont issues d'une réflexion des apprenants sur leur propre activité. Ceux-ci parviennent à transformer leurs interactions en un "schème d'action", ce "canevas des actions répétables " (Piaget, 1972), transposable dans de nouvelles activités, dans des contextes de travail différents, et en utilisant des outils ou logiciels variés. Cette extraction est activée lorsque les apprenants mettent en mots leur expérience au moment de la rédaction d'un "journal d'activité». Cet exercice rédactionnel permet aux apprenants d'auto-analyser leur activité réelle, à l'aide de questions dirigées. L'évaluation de ces acquisitions est rendue possible par l'analyse des traces des apprenants comme nous allons le voir dans la section suivante. 
Tableau 1: Plan d'un module de formation à la culture numérique

\begin{tabular}{|l|l|l|}
\hline \multicolumn{1}{|c|}{ Phases } & \multicolumn{1}{c|}{ Descriptions } & \multicolumn{1}{c|}{ Couches activées } \\
\hline I. de découverte & $\begin{array}{l}\text { prise en main d'un logiciel dédié } \\
\text { une pratique, fil directeur de la } \\
\text { formation }\end{array}$ & applicative \\
\hline 2. applicative & $\begin{array}{l}\text { comparaison des fonctionnalités } \\
\text { avec un autre logiciel pour la } \\
\text { même pratique }\end{array}$ & matérielle et politique \\
\hline 3. médiatique & $\begin{array}{l}\text { analyse des enjeux socio-économique } \\
\text { du média et comparaison avec un } \\
\text { média traditionnel }\end{array}$ & $\begin{array}{l}\text { analyse d'œurres tirées des arts } \\
\text { ou de la littérature numérique qui } \\
\text { illustrent les concepts abordés }\end{array}$ \\
\hline 5. artistique & $\begin{array}{l}\text { réflexion et connaissance des } \\
\text { aspects cognitifs ou ergonomiques : } \\
\text { étude comparée de productions liées } \\
\text { à la pratique étudiée. }\end{array}$ & sémiotique \\
\hline
\end{tabular}

\section{V. Évaluer l'apprentissage avec des traces d'activité}

Les expériences en présentiel que nous avons réalisées fournissent un ensemble de traces, notamment : les traces numériques involontaires produites par le dispositif d'interaction, les traces numériques volontaires qui concernent la production de l'apprenant, enfin les traces mnésiques qui désignent la mémoire que l'apprenant possède de son activité. Lorsque l'apprenant est confronté à ces traces, il renforce sa réflexivité, c'est-à-dire son esprit critique, et peut alors développer différents schèmes d'action et orienter son activité. Nous suivons ici les hypothèses sur le cours d'action de Theureau (2010). Dans ce dispositif pédagogique, nous avons ajouté un exercice supplémentaire pour renforcer cette réflexivité, c'est la rédaction d'un journal d'activité qui permet d'augmenter l'effort d'explicitation de l'apprenant de son activité. L'étude de ce journal permet aussi d'évaluer cet effort réflexif. Son analyse a fait l'objet d'une étude qualitative. Nous avons tout d'abord établi une liste des « formes de réflexivité » en nous appuyant sur la proposition de Derobertmasure et Dehon (2009) qui identifie onze de ces formes. Nous avons ensuite repéré ces formes dans les journaux d'activité en les marquants à l'aide du logiciel R-QDA ${ }^{12}$. Nous avons alors repéré d'autres formes d'analyse des apprenants, qualifiées d'« objectives » puisqu'elles concernent des analyses de l'apprenant qui portent sur les contraintes du dispositif extérieur d'écriture, et non pas sur leur propre activité. L'ensemble de ces formes ont fait l'objet d'une représentation de type ontologie, qui apparaît dans la figure 
Figure 3 : Schéma pour l'ontologie des formes réflexives et objectives.

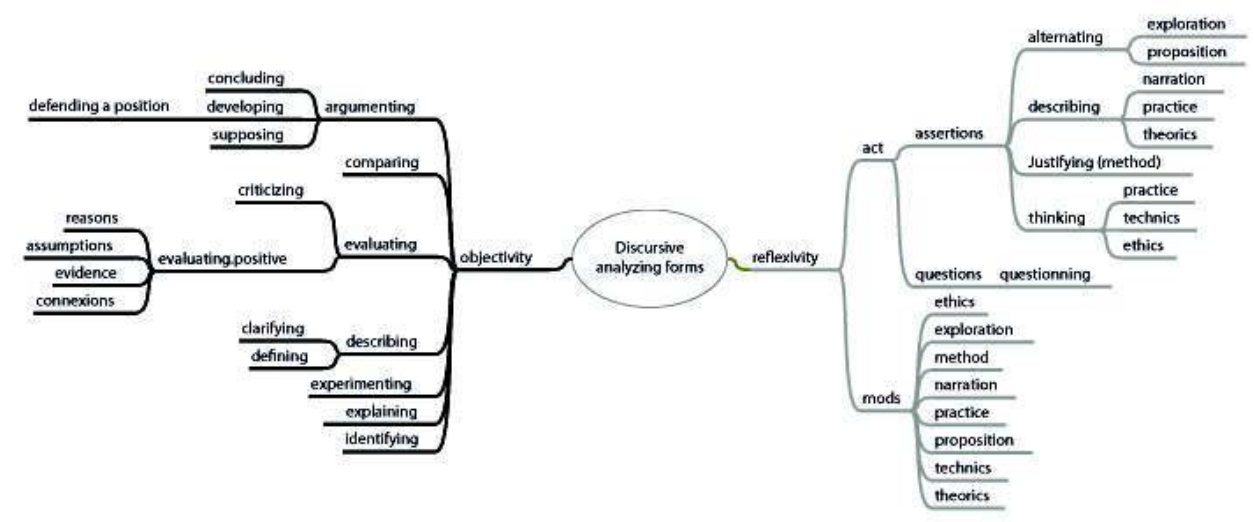

21 Nous avons réalisé l'analyse des discours des apprenants en repérant les «mondes lexicaux » conversationnels dans les journaux d'activité. L'analyse des similitudes met en évidence les réseaux lexicaux à l'aide du logiciel Iramuteq ${ }^{13}$. La figure 2 montre le graphe des similitudes établi lors de l'analyse des journaux d'activité produits pendant la formation «écriture sur un réseau social " par douze étudiants à Béziers en situation d'échec à l'IUT. Nous leur avons donné comme consigne de réaliser un document multimédia suite à la visite du musée des Beaux-Arts de la ville. Ils ont alors répondu à des questions ouvertes dans un "journal d'activité». Cet outil a pour but à la fois d'évaluer les processus cognitifs qu'ils ont mobilisé, et de leur permettre de renforcer leur réflexion à propos des outils abordés. Les questions sont structurées selon le plan précédemment exposé.

22 Ce graphe montre notamment l'emploi séparé de notions abstraites (information, réseau social) par rapport à des notions plus concrètes comme "Facebook». Cet effort d'abstraction est symptomatique d'une volonté de prise de recul des apprenants sur leur activité.

Nous avons mesuré la part des formes de réflexivité et d'objectivité dans les discours des apprenants à l'aide de marqueurs qui identifient certains segments de discours. Ces formes sont donc mesurables en nombre et quantifiable en nombre de mots et de caractères. La comparaison des formes de réflexivité $(42 \%)$ et des formes d'objectivité (58 \%) montre l'importance des formes de réflexivité. Les formes d'objectivité ont cependant besoin de plus de mots pour s'exprimer : les formes d'objectivité représentent $35 \%$ des caractères contre $65 \%$ pour les formes de réflexivité. 
Figure 4 : Graphe des similitudes, formation « écriture sur un réseau social ».

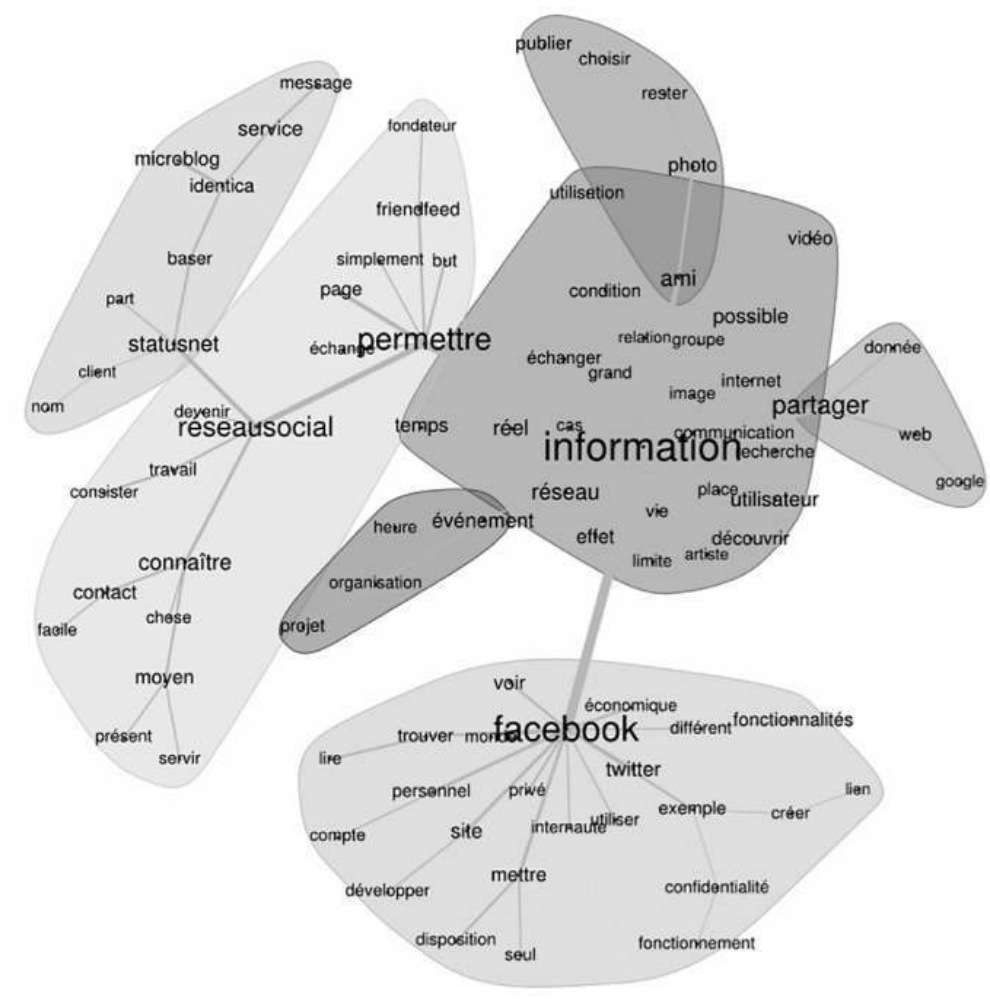

\section{Perspectives pour la plateforme sémantique}

Les expériences réalisées en présentiel montrent la pertinence de ce modèle pour renforcer la réflexivité des apprenants lorsqu'ils utilisent des outils numériques. Le passage du présentiel vers le distanciel suppose la mise en œuvre de critères d'évaluation différents, plus quantitatifs. Une première étape a été réalisée avec notre contribution au projet Arbradoc, plateforme de formation à distance à la recherche documentaire et à la culture numérique ${ }^{14}$. Nous avons fourni les contenus sur la culture numérique à partir de l'approche présentée ci-dessus. On peut voir une copie d'écran du projet en ligne sur la figure 3. 
Figure 5 : Formation en ligne à l'écriture collaborative (Arbradoc).

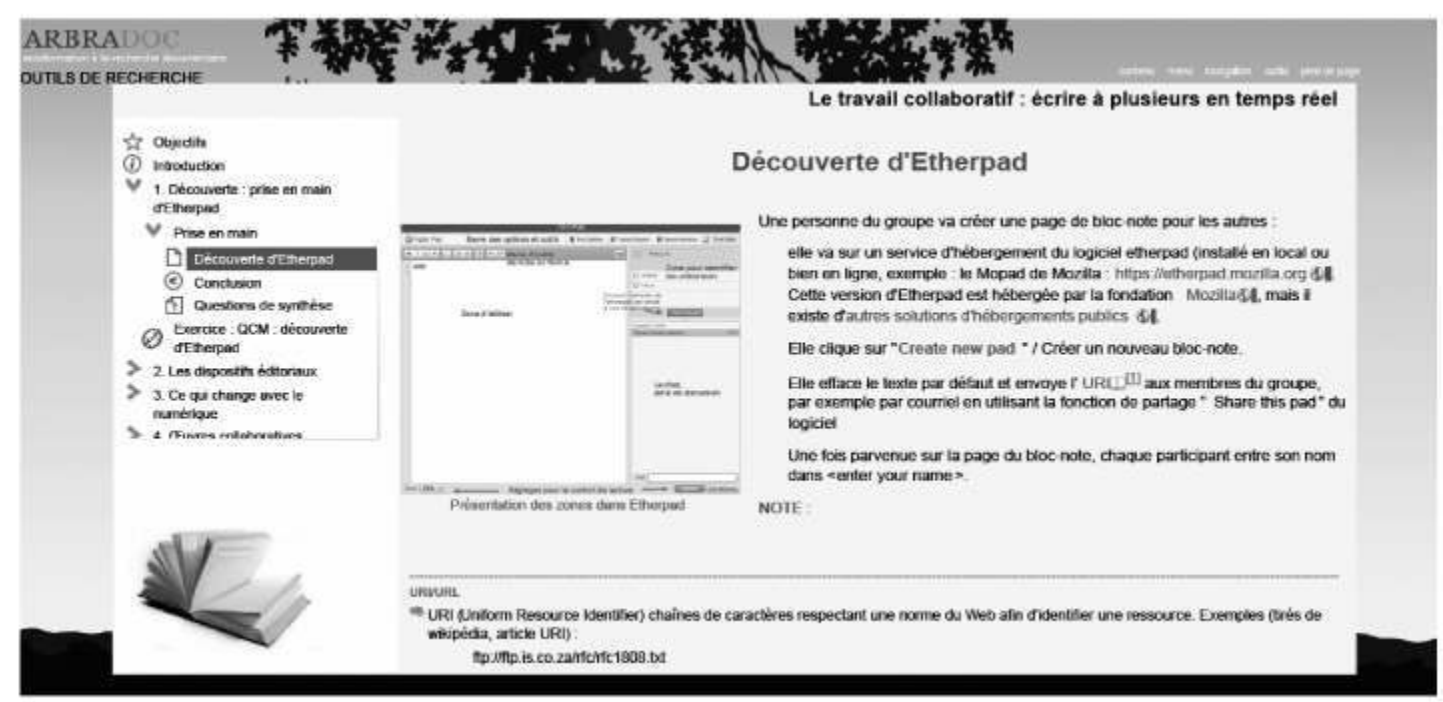
compétences numériques acquises par réflexion sur l'activité numérique, ce qui requiert d'autres protocoles d'observation. En outre, il est intéressant d'étudier les traces d'interaction dans le dispositif d'enseignement à distance pour améliorer la qualité du dispositif. Dans une plateforme à distance, il est possible de récolter des traces numériques et de concevoir divers indicateurs. Parmi ceux-ci, on relèvera: le niveau de satisfaction des utilisateurs par type d'utilisateur (enquêtes quantitatives et qualitatives), le nombre de ressources accessibles à travers cette plateforme dans le cas d'une approche collaborative; le nombre et la durée des interactions avec ce système en fonction des profils des utilisateurs, apprenant ou formateur.

\section{Conclusion}

Les technologies du Web sémantique assurent une meilleure accessibilité des ressources et une visibilité accrue sur le Web. Elles permettent aussi d'expliciter, d'éclaircir et de structurer les situations d'apprentissage elles-mêmes. Le modèle humain/machine en couches fournit le socle sémantique à partir duquel décrire de façon fine les schèmes et concepts en jeu dans l'activité complexe. Il est bâtit ni sur une hétérogénéité ni sur une fusion entre les concepts d'activité humaine et d'activité technique, mais sur leur articulation. Au final, c'est l'effort d'analyse réflexif, critique et collaboratif des apprenants et des formateurs qui fournira des conditions très favorables à la mise en œuvre d'une formation numérique de qualité. 


\section{BIBLIOGRAPHIE}

Ausubel, (David P.), (1960), « The use of advance organizers in the learning et retention of meaningful verbal material », in Journal of Educational Psychology, $n^{\circ} 51$ (5), 267-272.

Berners-Lee, (Tim) ; Hendler, (James) et Lassila, (Ora), (2001), « The semantic web », in Scientific american, $n^{\circ} 284(5), 28-37$.

Beziat, (Jaques), (2012), « Former aux TICE : entre compétences techniques et modèles pédagogiques ", Revue internationale des technologies en pédagogie universitaire, vol.9, n¹-2, 53-62.

Bouchardon, (Serge) ; Cailleau, (Isabelle) ; Crozat, (Stéphane) ; Bachimont, (Bruno) et Hulin (Thibaud), (2010), « Explorer les possibles de l'écriture multimédia », in Les Enjeux de l'information et de la communication, $n^{\circ} 1$.

Delamotte, (Éric) ; Liquete (Vincent) et Frau-Meigs (Divina), (2014), « La translittératie ou la convergence des cultures de l'information : supports, contextes et modalités », in Spirale, $n^{\circ} 53$, 145-156.

Derobertmasure, (Antoine) et Dehon, (Arnaud), (2009), « Vers quelle évaluation de la réflexivité en contexte de formation initiale des enseignants? ", in Questions Vives, $n^{\circ} 6(12), 29-44$, décembre.

Deuze (Mark), (2006), « Participation, remediation, bricolage : Considering principal components of a digital culture ", in The information society, n²2(2), 63-75.

Dicheva, (Darina) ; Sosnovsky (Sergey) ; Gavrilova (Tatiana) et Brusilovsky (Peter), (2005), "Ontological web portal for educational ontologies », in Proceedings Of Applications of Semantic Web Technologies for E-Learning Workshop, (SW-EL'05 - in Conjunction with 12th Int. Conf. on Artificial Intelligence in Education AI-ED 2005), Amsterdam, pp. 19-29.

Engeström, (Yrjö), (1999), « Activity theory et individual et social transformation », in Engeström (Yrjö) ; Miettinen (Reijo) et Punamäki (Raija-Leena), éditeurs, Perspectives on activity theory, pp. 19-38, New-York : Cambridge University Press.

Foucault, (Michel), (1994a), «L'écriture de soi », texte n³29, in Dits et Écrits, 1954-1988, tome IV : 1980-1988, pp. 415-431. Paris : Gallimard.

Foucault, (Michel), (1994b), « Le jeu de Michel Foucault », texte n²06, in Dits et Écrits, 1954-1988, tome III : 1976-1979, pp. 238-329, Paris : Gallimard.

Gruber, (Thomas. R.), « A translation approach to portable ontology specifications ", in Knowledge acquisition, $n^{\circ} 5(2), 199-220$.

Manovitch, (Lev), (2002), The language of new media. Cambridge : The MIT Press.

Pelissier, (Chrysta) et Mailles-Viard Metz (Stéphanie), (2012), « Le C2i en IUT : une aide aux acteurs impliqués ». Questions Vives. Recherches en éducation, n¹7(7), 105-122.

Piaget, (Jean) et Inhelder (Bärbel), (1972), The psychology of the child. New-York: Basic Books.

Raskin (Robert G.) et Pan (Michael J.), (2005), « Knowledge representation in the semantic web for earth et environmental terminology (SWEET) », in Computers \& geosciences, $n^{\circ} 31$ (9), 1119-1125.

Simondon, (Gilbert), (1969), Du mode d'existence des objets techniques. Paris : Aubier-Montaigne. 
Stiegler, (Bernard), (2008), Prendre soin : De la jeunesse et des générations. Paris : Flammarion.

Theureau, (Jacques), (2010), « Les entretiens d'autoconfrontation et de remise en situation par les traces matérielles et le programme de recherche "cours d'action" ", in Revue d'anthropologie des connaissances, $n^{\circ} 4(2)$, 287-322.

UNESCO, Convention pour la sauvegarde du patrimoine culturel immatériel, http:// unesdoc.unesco.org/images/0013/001325/132540f.pdf [consulté le 15/09/2014], 17 octobre 2003.

Vygotsky (Lev), (1997), Pensée et langage. Paris : La dispute.

Willerman (M.) et Mac Harg (R. A.), (1991), « The concept map as an advance organizer », in Journal of Research in Science Teaching, $n^{\circ} 28(8), 705-711$.

Zimmermann (Hubert), (1980), « OSI reference model-the ISO model of architecture for open systems interconnection ", in Communications, IEEE Transactions, n²8(4), 425-432.

\section{NOTES}

1. Massive Open Online Courses.

2. Friend of a friend : vocabulaire permettant de décrire des personnes et les relations qu'elles entretiennent entre elles.

3. Semantic Web for Earth and Environemental Terminology http://sweet.jpl.nasa.gov/ontology.

4. Sharable Content Object Reference Model.

5. Learning Object Metadata.

6. PCIE, dit aussi International Computer Driving Licence http://www.pcie.tm.fr

7. TOSA http://www.isograd.com/FR/index.php

8. CISD http://www.cambridge-vellum.gr/newsite/

9. C2I http://www2.c2i.education.fr/

10. Open Systems Interconnection. Il s'agit d'un modèle de communications entre ordinateurs proposé par l'Organisation internationale de normalisation (ISO).

11. http://thibaud.hulin.free.fr/precip_foad

12. http://rqda.r-forge.r-project.org

13. http://iramuteq.org/

14. http://arbradoc.bu.univ-paris8.fr

\section{RÉSUMÉS}

Les technologies du Web sémantique pour l'éducation ont plusieurs atouts : accessibilité et pérennité des ressources, explicitation et organisation accrues. Nous présentons ici un projet de plateforme sémantique, l'intérêt de ces technologies, ainsi qu'un modèle de la communication qui articule sans les confondre les activités de l'humain et de la machine. Celui-ci a pour but d'aider apprenant, formateur et théoricien à mieux penser, de manière réflexive et collaborative, les activités de chacun. Les réalisations présentées à propos de la culture numérique ouvrent la voie vers une formation numérique de qualité. 
The technologies of Semantic Web for education have several advantages: accessibility and sustainability of resources, increased clarification and organization. Here we present our project of a semantic platform, the interest of those technologies, and a human / machine communication model that helps learner, teacher and scientist to think better, reflexively, the activities of each of them. The experimentations about digital culture pave the way for a quality digital training.

INDEX

Mots-clés : communication, culture numérique, formation, web sémantique

Keywords : communication, digital literacy, e-learning, semantic web

\section{AUTEUR}

THIBAUD HULIN

Maître de conférences, Université de Bourgogne 